\title{
New Members
}

DERManent membership records have been kept only since 1946-so in this listing members joining during the period Jan. I-Apr. I, I948, are called "new" if they did not belong in either 1946 or 1947 . Because of various factors there may be an unusual degree of error in this first listing. We would appreciate your help in making our records accurate. So would you please notify N. Orwin Rush, Executive Secretary, 50 E. Huron St., Chicago I I, of any corrections which should be made. New institutional members will appear in a later listing.

Ackley, Mrs. Corinne B., University of Washington Adams, Dorothy Jeanette, Riverside, Calif., Central Junior High

Adams, Florence Elizabeth, Yale University

Aguayo, Jorge, Universidad de la Habana

Alexander, Leona May, Oakland, Calif., Public Library

Alexander, Virginia, University of South Carolina

Alford, Attie A., Florida State University

Alford, Ruth Virginia, University of Delaware

Amesse, Helen M., Unniversity of Denver

Anders, Richard Lear, Champaign, Illinois

Anderson, Geraldine D., Pittsburgh Consolidation Coal Co.

Anderson, Isabelle T., University of Utah

Anderson, Mary Agnes, Belhaven College

Anglemyer, Mary, New York State Division of Placement and Unemployment Insurance

Appell, Alice Jacquette, University of Illinois

Archer, Leonard B., Jr., Detroit Public Library

Armantrout, Collean S., Army Map Service

Armbrister, Roberta F., David Taylor Model Basin

Armstrong, Clifford R., State College of Washington

Armstrong, D. G., Calif. Institute of Technology

Arpke, Erna Fredericke, University of California, Berkeley

Asterholdt, Aileen, Lenoir Rhyne College

Averill, Elizabeth A., Joslyn Memorial Art Museum of Omaha

Averitt, Edith, Chapel Hill, North Carolina

Averitt, Ruby Elizabeth, Texas State College for Women

Bach, Harry, University of Oregon

Bacon, Vera J., University of Iowa

Bailey, Dorothy, McCormick Theological Seminary

Bailey, Joe Handen, Murray, Kentucky, State Teachers College

Bailey, Lucille E., Hunter College

Bailey, M. Lois, Northern State Teachers College, Aberdeen, South Dakota

Baird, Mrs. Violet M., Southwestern Medical College

Baker, Mrs. Mabel Peterson, Boise Junior College

Baker, Mrs. Mary W., Chicago Natural History Museum

I3aldwin, Ruth Marie, San Jose, Calif., State College

Balfour, Greta H., Catholic University of America

Ball, Phyllis, University of Arizona

Ballou, Hubbard W., University of Illinois

Banay, George L., Worcester, Mass., State Hospital

Bane, Suda Lorena, Stanford University

Banwell, Katharine E., California State Library

Barber, Constance Roberta, Howard University

Barden, Bertha R., Western Reserve University

Barham, Elizabeth'J., Catholic University of America

Barksdale, Gaynelle W., Atlanta University

Barrett, Elizabeth, Toledo Public Library

Barrett, Marguerite E., Creighton University

Barrnes, Vera Mae, Grinnell College

Barron, Mrs. Kathleen M., Georgia State Dept. of Public Health

Bartlett, Eleanor Louise, Massachusetts Institute of Technology

Bartlett, Helen, Parsons Junior College

Bartolini, R. Paul, University of Wichita

Bassett, Mary Henrian, Southern Christian Institute

Batten, Charles E., Crozer Theological Seminary

Baxter, Mabel G., Kansas State Library

Baxter, Marie Winslow, Union College (Barbourville,

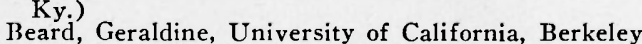

Beckham. Jessie E., Grand Rapids Junior College

Beem, Miss Blake, University of Louisville
Bell, Bernice K., Wesley Junior College

Bellingham, Harold, University of Denver

Bennett, Melvin, Kent Library (Cape Girardeau, Mo.)

Bentz, Dale M., East Carolina Teachers College (Greenville, N.C.)

Berg, Virginia A., University of Illinois

Bevis, Leura Dorothy, University of Washington

Bielby, Ruth M., Syracuse University

Billington, Donna Jean, Harris College of Nursing

Bishop, Amie-Louise, University of Colorado

Bishop, Mrs. Ethel Langdon, Nebraska Wesleyan University

Bishop, H. E., U. S. Army Medical Library

Bitting, Barbara, University of Illinois

Blackburn, Robert Harold, University of Toronto

Blackie, Marian Lois, University of California, Berke-

Blair, Naomi B., Indiana University

Blake, Nelson M., The National Archives

Blakiston, Joan Madeline, Wellesley College

Blazier, George J., Marietta College

Bleyhl, Norris Arthur, Mesa College

Bliss, Helen, Eastern Oregon College

Bobbitt, Margaret S., Marshall College

Bond, Elizabeth M.,' Minneapolis Public Library

Borden, Elmira, Dayton Public Library

Borden, Fanny, Vassar College

Borden, Joseph C., University of Arkansas

Bourneuf, Mother Aimee C., Manhattanville College

Bouwhuis, L. Andrew, Canisius College

Bowers, Constance C., Champlain College

Boyce, George Kenneth, Pierpont Morgan Library

Boyd, Alice L., University of Illinois

Boysworth, Willa M., Huntingdon College

Bradley, Mary, River Falls, Wisconsin, State Teachers College

Branch, Vivian, University of Georgia

Branham, Alice Irene, Minneapolis Public Library

Breedlove, Stella, U. S. Dept. of Agriculture, Plant Industry Sub-Branch, Beltsville, Md.

Brett, William Howard, University of California, Berkeley

Bright, Franklyn Furman, Brown University

Brinkler, Bartol, Harvard College

Britton, Jasmine, Los Angeles City Schools

Brown, Flora M., Minneapolis Public Library

Brown, Janet E.. University of Buffalo

Brown, Robert B., University of Michigan

Brown, Susie Persons, John B. Stetson University

Brownlee, Mrs. Lenore M., Adams State College (Alamosa, Colorado)

Brubaker, Hastings Alton, Lawrence College

Buchholz, Marion, Buffalo, New York State College for Teachers

Buck, Gertrude, Manhattanville College of Sacred Heart

Buck, Kathryn L., Chadron, Nebraska State Teachers College

Buffum, Charles Walbridge, Library of Congress

Bullock, Mary Lou, Pennsylvania State College

Burge, Dorothy E., American Institute for Foreign Trade

Burgess, Virginia Leslie, U. S. Office of Education

Calmbach, Elinor, Southwestern Medical College

Cam, Gilbert A., New York Public Library

Camacho, Blase, W. R. Farrington High School Library (Honolulu 35, Hawaii)

Campbell, Ella M., University of Michigan

Campbell. Marilyn Ida, Columbus Public Library

Canny, Robert A., Denver Public Library

Carabelli, Angelina J., U. S. Dept. of Agriculture 
Carbutt, Mrs. Katharine K., Los Angeles Public Library Carleton, Florence Mildred, Wayne University

Carlson, Bern M., Golden Gate School of Management

Carlson, Ruth A., Augustana College

Carnes, Mrs. Frances Cox, Westminster College

Catey, Cieorgia E., University of California, Los Angeles

Cavanaugh, Mrs. Joyce J., Joint Reference Library (Chicago)

Cavenaugh; Lucile C., St. Francis College

Challenger, Helen Field, Los Alamos Library

Chandler, Louise F., Massachusetts College of Phar macy

Charvat, Beatrice, Cleveland, Ohio

Chidekel, William, New York University

Chism, Harriet Jean, Washoe County (Nevada) Li brary

Clayton, Frances, Texas College of Mines and Metallurgy

Clark, Annie Ree J., New Rochelle Public Library

Clay, Wafa F., Mount Airy, Md.

Clayton, Herbert Vincent, California State Library

Cleckner, Marie E., Library of Congress

Clemence, Stella Risley, Library of Congress

Cleveland, Hazel E., Western Michigan College of Education

Clugston, Katharine W., Library of Congress

Coblentz, Ruth Irene, Manchester College

Coffin, Lewis Charles, Library of Congress

Cohen, Joseph Lorence, Rutgers University

Colcord, Mabel, Entomology \& Plant Quarantine, Washington, J).C.

Coleman, Laura Marion, New Jersey College for Women

Collins, Eleanor Stuart, University of Michigan

Collins, Eugenia A., Judson College

Collins, Wallace Clifford, University of New Zealand

Colson, Elizabeth, Armstrong Junior College

Colvin, Laura Catherine, Simmons College

Coninx, Ardis Dechman, Evanston Community College

Conklin, Robert J., Springfield College

Connor, John Michael, Washington, D.C.

Conrad, Agnes C., University of California, Los Angeles

Conrad, Rev. Simon R., St. Fidelis Seminary

Converse, Helen C., Virginia Polytechnic Institute

Cooksley, Mrs. Florence A., Washington, D.C

Cooley, Ëleanor Graham, Berwyn. R.F.J., Md.

Cooper, Dorothy M., University of Washington

Cooper, Evelyn L., Glendale (California) Public Library

Cope, Barbara Jean, University of California, Los Angeles

Corbin, Phyllis May, Potsdam (New York) State Teachers College

Costello, Jora H., Oregon State College

Covalesky, Eleanor Eunice, American National Red Cross, Washington, I.C.

Cowgill, Lois, Oberlin College

Craig, Alice L., North Jakota Agric. College

Cramer, Dorothy Mildred, National Institute of Health

Creekmore, Pollyanna, Lawson McGhee Library

Crenshaw, Esther Mary. Wílberforce, Ohio, College of Education and Industrial Arts

Cronin, John William, Library of Congress

Crutcher, Florence Jennings, St. Louis Public Library

Currier, Margaret, Harvard University

Curtiss, Dorothy W. Wells College

Custer, Benjamin Allen, Detroit Public Library

Cutlip, Mary E. U. S. Naval Powder Factory, Indian Head, Md.

Dafoe, Elizabeth. University of Manitoba

Dahl, Carl, R. R. Donnelley and Sons Co. Printers, Chicago

Damgard. Mrs., Detroit Public Library

Dana, D. Brownell, Ripon College

Dana, Virginia, Knox College

Danielb, Lorna M., Harvard Business School

Dauphinee, Bede Ä., Siena College

Davis, Lillian C., Florida A \& M College

Davis, Nannie Jo, Jacksonville, Alabama State Teachers College

Jay, Katherine Bolt, Duke University

Dean, Mrs. Maud L., John and Mabel Ringling Museum of Art

DeRenne, W. W., University of Georgia de Tirefort Mrs, Magdelaine, Amherst College DeVolder, Arthur Leon, Tacoma Public Library DeVore, Helen Lawton, Evans Signal Laboratory, Long Branch, N.J.

Dickson, Janet S., University of Iowa

Dimock, Fred L., University of Michigan

Dix, William S., The Rice Institute

Dolan, Philip Hilary, Harvard College

Dondy, Emanuel, Dept. of Agriculture

Doolittle, Elizabeth Whiting, Plymouth (New Hampshire) Teachers College

Dover, Leta Sowder, Bacone College

Downey, Bernard Francis, Jr., Harvard University

Dozier, Lois G., University of Notre Dame

Drewry, Lucie T. M., Virginia Polytechnic Institute

Drozda, Raymond J., Armour and Co. Patent Library, Chicago

Dugan, Mrs. Hammond J., University of Kentucky

Dunlap, Joseph R., City College of New York

Dunlap, Mollie E., Wilberforce, Ohio, College of Education and Industrial Arts

Dunten, Bernice L., Purdue University

DuPre, Mary S., Wofford College

Dwelle, Alberta Barbara, Alderson-Broaddus College

Jyer, Lila N., Our Lady of the Lake College

Earle, Elinor S., Lincoln Library, Springfield, Illinois

Earley, George Edward, Boston Public Library

Eaton, Thelma, Washington, D.C.

Edwards, V. W., Edwards Brothers, Inc., Ann Arbor, Michigan

Eileen, Sister M., Caldwell College

Ekstrand, Margaret L., Eastern Illinois State College

Eldredge, Lucile Mildred, Yankton College

Elliott, Evelyn, University of Washington

Elliott, L. R., Southwestern Baptist Theological Seminary

Ellis, Helen Harriet, Detroit Public Library

Ellis, Sidelle B., Clemson College

Elvira, Sister Mary, College of Saint Francis

Fndelman, Theodore, Santa Maria Junior College

Enders, Gertrude E, U. S Civil Service Commission

Entrikin, Isabelle Webb, Army Medical Library

Erkert, Clayton A., University of Pennsylvania

Eulass, Elizabeth M., The Viking Press, Inc

Evans, Hazel, Cedar Rapids Public Library

Evans, Miss Kennedy, Texas State College for Women

Evans, Luther H., Librarian of Congress

Ewen, Florence S., Oregon State College

Ewing, William S., University of Michigan

Farrell, Colman Joseph, St. Benedict's College

Featherly, Lucia C., Gary Law Library, Chicago, Illinois

Feehan, Wm. H., Maryland Historical Society

Fehner, Cornelia Maria, University of Southern California

Felland, Nordis, American Geographical Society

Fenner, Hilda Hisey, Madison College

Ferguson, Priscilla Just, University of California, Berkeley

Ferren, Dorothy Adele, Seventh-day Adventist Theological Seminary

Ferring, Geraldine, Napa Junior College

Fields, Donald E., Lebanon Valley College

Finder, Jane, A.L.A. Headquarters

Finney, George J., Dept. of the Army, Civil Affairs Division

Finney, Jeannette E., Elizabeth McCormick Memorial Fund, Chicago

Fisher, Gwendolyn, Lyons Township High School and Junior College

Fletcher, Charlotte Goldshorough, St. John's College

Floyd, Lawrence C., Columbia University Library School

Ford, Carolyn W., Tuskegee Institute

Forsberg, Helen M., University of Washington

Francis, Kathleen Burke, University of Richmond

Francis, Roger B., New York Public Library

Frank, Emma Lucile, Oberlin College

Franks, Mary Loretta, University of Idaho

Fraser, Bessie Fairbairn, Presbyterian College

Fraser, C. C., Detroit Public Library

Fraser, M. Doreen, University of British Columbia

Friedrich, Mrs. Esther B., Pennsylvania State College

Fritchman, Mary Louise, Pratt Institute

Gallagher, Agnes, Harvard University

Gallaway, Margaret, University of Arkansas 
Galloway, Mary Elizabeth, Freed-Hardeman College Gambee, Budd L., University of West Virginia

Gambuto, Lucille E., Brown University

Ganfield, Jane, Purdue University

Geheren, Margaret E., St. Thomas College

Genung, Marie D., Mills College

Giles, Eloise, American Federation of Labor, Washington, D.C

Gillette, Fredericka Botsford, University of Michigan

Gillingham, Frank Truscott, Crozer Theological Seminary

Gilmore, Rev. Lawrence Blair, Reformed Episcopal Theological Seminary

Gilmore, Sylvia, University of Illinois.

Giordina, Angela, Pace Institute

Githens, Alfred Morton, Mt. Vernon, New York

Gnoza, Edmund, University of Oregon

Goettel, Harold M., Academy of Medicine of Northern New Jersey

Goldsmith, Gertrude, Detroit Public Library

Goode, M. Harriet, Detroit Public Library

Goodman, Martha, Youngstown Public Library

Gould, Mabel P., University of Iow a

Gould, Marie L., U. S. Dept. of Agriculture, Portland, Oregon

Gourlay, John Wallace G., Indiana University

Graham, Cornelia Ayer, Clemson College

Graham, Kathryn, Galesburg Branch, University of Illinois

Grainger, William Keith, Compton Junior College

Graves, Mrs. Louise Harper, Georgia Baptist

Gray, Ruth M., Northern Baptist Seminary

Green, Mrs. Jean Mitchell, University of Pennsylvania

Greenfield, Kathleen Elizabeth, Schenectady Public Library

Greenwood, Walter Bart, Library of Congress

Gribbin, John Hawkins, University of Missouri

Grimmett, Mrs. Mary E., Newton D. Baker V. A

Center, Martinsburg, W.Va.

Groesbeck. Joseph, Detroit Public Library

Groves, May M., Oklahoma Agric. \& Mechanical Col. lege

Gruen, Sonia L., New York, New York

Hale, Ruth E., University of Washington

Hambrick, Thera Ollis, Georgia State Woman's College

Hamilton, Carl Wessberg, University of Denver

Hamilton, Chas. G., Library of Congress

Hamlyn, Mary Grace, McGill University

Hammend, Dorothy S., Iowa State College

Hamrick, Lillian Aline, Library of Congress

Hansen, Oda Bali, University of Michigan

Harper, Mrs. Mildred M., Yerkes Observatory

Harper, Mrs. Thelma G., Wilberforce, Ohio, College of Education and Industrial Arts

Harrell, Rena C., Queens College

Harris, Virginia, McPherson College

Harrison, Alice Lucille, University of Michigan

Hartkopf, Margy Alberta, Clemson College

Hauck, Celia R., Marquette University

Hauck, Helen G., Rensselaer Polytechnic Institute

Haughn, Myrtle Mary, Citrus Experiment Station, Riverside, Calif.

Hausle, Ruth E., Washington, D.C.

Hayes, Margaret, University of Denver

Haynes, Virginia Priest, Evansville College

Hebert, Mary Alice, Louisiana State University

Hedstrand, Lillian, New York State College of Agri culture

Heeth, Louisa Varnedoe, Florida State University

Hefling, Helen, University of New Mexico

Heicke, Dorothy Elizabeth, Southern Illinois University

Hemens, Rollin D, University of Chicago Press

Hendricks. King, Ûtah State Agricultural College

Hennig, Mrs. Winifred St. John, Wellesley College

Herling, John P. New York Public Library

Hettich, Ernest L., New York University

Hexner, Gertrude, Washington, D.C.

Heyer, Ann Harriet, North Texas State Teachers College

Hicks, Mary Elizabeth, University of Maryland

Higgs, E. Lucille. Meredith College

Higley, Mrs. Lutie Lee, University of Arizona

Hill, Gertrude Frances, University of Arizona

Hill, Gertrude Frances, University of Arizona

Hill, Mildred M., Washington State Lib

Hitchcock, Carrol Doane, Triple Cities College of Syracuse University
Hitchcock, Jessie C., University of Minnesota

Hoffman, Kathleen E., A.F.S.S.S. Library, Craig Field, Selma, Alabama

Hofmaester, Mrs. T. M., Art Institute, Chicago, Illinois

Hogg, Elizabeth Olin, University of Chicago

Holcomb, Laurel E., University of Illinois

Holder, Elizabeth Jerome, Woman's College, University of North Carolina

Holland, Mrs. Sarah B., Tulane University

Holman, Emma Linton, Mary Baldwin College

Holmes, Donald C., Library of Congress

Holt, Grace Frances, Smith College

Hooker, Mrs, Ruth H., Naval Research Laboratory, Washington, D.C.

Hooper, Blanche H., Tufts College

Hoven, Earl Emery, Madison, Wisconsin

Howard, Valerio Supearl, Tallahassee, Florida, Carnegie Library

Howe, Harriet E., University of Denver

Hoyle, Nancy E., F. E. Compton Co.

Hughes, Jessie Hopwood, Ashland Junior College

Hulbert, James Alexander, Virginia State College

Hulbert, Mabel Irene, Michigan College of Mining and Technology

Huston, H. N. Dorothy, Madison (Wisconsin) Free Library

Inez, Sister Marie, The College of St. Catherine

Irvin, Charles Edgar, University of Miami

Irvine, Kate Tipton, University of Kentucky

Jacks, Beulah B., U. S. Navy Laboratory, San Iiego

Jackson, Ellen, University of Colorado

Jackson, Eugene Bernard, Air Materiel Command, Wright Field, Dayton, Ohio

Jackson, Ruth Whitlock, Air Materiel Command, Wright Field, Dayton, Ohio

James, Alice, Creole Petroleum Corp., Caracas, Venezuela

Jamison, Louise, University of Corpus Christi

Jeeck, Elsa L., University of Wisconsin Extension Division

Jennings, John Melville, Virginia Historical Society

Jennings, Laura Louise, Cornell University

Johns, Loeta Lois, University of Washington

Johnson, Bessie Margaret, University of Michigan

Johnson, Elmer Douglas, Limestone College

Johnson, Lacie M., North Carolina A \& T College

Johnson, Louise C., Rockford Public Library

Johnston, Margaret Young, Lockwood Memorial Library, Buffalo

Jones, Elizabeth Burch, Princeton University

Jones, Harold Degner, Fairmont, West Virginia, State College

Jones, Sarah Frances, Emory University

Joseph, Sister M., Rivier College

Joyce, Phyllis Bray, Rhode Island State College

Kaestle, Sarah Elam (Mrs. Karl), Goodwyn Institute

Kahler, Doris Lucille, East Orange Public Library

Kanable, Sara Marjorie, Pembroke (North Carolina) State College

Karpenstein, Katherine, Standard of California, San Francisco

Kelley, Edna Irene, Illinois State Normal University

Kelly, Frances H., Carnegie School of Technology

Kemp, Erle Pilcher, Citv College of New York

Kemp, Virginia, Baylor University

King, Dorothy, Smith College

Kirwan, Alice Stokes, Eleventh Naval District Head. quarters

Kistler, Delilah E., P.A.C.C. Rittenhouse College

Kistler, Ellen Downey, South Bend Public Library

Kneil, Gertrude M., Unniversity of Pittsburgh

Knight, Mrs. Hattie M., Brigham Young University

Knox, Margaret E., Úniversity of Illinois Library School

Koenig, Rev. Harry Corcoran, St. Mary of the Lake Seminary

Komier. Helena Craig, Ứniversity of Virginia

Kopp, Emma Lue, Purdue University

Koster, Claire Desmong, Florida State University

Kovanda, Anne, Illinois Geological Survey

Kramer, Sidney, Washington, I).C.

Krenitsky Michael Von, Texas Military College

Kruger, Charles F., Regis. College

Kruse, Mildred M., Jersey City, New Jersey State Teachers College

LaHue, Katherine M.. Colorado Woman's College

Lanning, Roland J., Üniversity of British Columbia 
Lee, Kathryn Ann, A.L.A. Headquarters

Lee, Margaret I., Columbia University

Lejeune, Eugenia Dickson, Marine Corps Schools, Quantico, Virginia

Lester, L. Alma, Florida State University

Levesque, Elaine M., U. S. Dept. of Agriculture, Washington, D.C.

Levins, Hazel Kirk, Mutual Benefit Life Insurance Co.

Lewis, Alice Martha, Worthington, Minnesota, Public Schools

Lewis, Eleanor F., Northwestern University

Lewis, Esther, New York Public Library

Lewis, Katharine Wendell, University of Louisville

Lewis, Leora J., F. E. Compton and Co.

Lewis, Samuel F., University of Michigan

Libbey, David C., State College of Washington

Linton, Howard Philip, Columbia University

Lockhead, John L., The Mariners' Museum Library

Loser, Arla Lorraine, Illinois Department of Labor, Chicago

I.owe, Frances Somers, Georgia School of Technology

Lowe, Mrs. Velma Singleton, Nashville, Tennessee, $A$ and I State College

Lowenthal, Mrs. Ruth H., Fort Hays, Kansas, State College

Lucas, Aileen, Fairmont State College (West Virginia)

Lumbard, Emily Charlotte, University of California (Berkeley)

Lunn, Jean, Fraser Institute

Lyman, W. Newlin, Minnesota Bookstore (Minneapolis)

Lyons, John Frederick, McCormick Theological Seminary

McCameron, I.loyd, University of Texas

McCarthy, Cecelia M., University of Illinois

McClelland, Marybelle, Iowa State Teachers College

McConagha, Iva, Muskingum College

MacConomy, Edward Nelson, Library of Congress

McElderry, Stanley, Chicago, Illinois

McGordy, Emily L., Wilmington Institute Free Library

Macgowan, Margaret, Los Angeles City College

McGrath, Alice V., Pasadena Public Library

Mack, James D., Lehigh University

Mckechnie, Agnes Mary, University of Denver

McKillop, Marjorie Leslie, Istanbul Woman's College

Mcknight, Eugene C., Riverside College

MacLean, Helen Hirt, Chase College

McNernev, Charles Thomas, Jr., University of New Hampshire

McTighe, Marianne, University of Scranton

Maddox, Eugenia, University of Tulsa

Marguerite, Sister Mary, Dominican College

Marshall, Elizabeth B., Cleveland College

Marshall, Shelley Virginia, Pacific Union College

Marshall, Mrs. Tommie M., Camphellsville College

Martin, Adele Bernice, Southern University (Louisiana)

Martin, Mrs. Ethel Garey, Dartmouth College

Martinciell, Diane, Westminster Choir College

Martini, Mrs. Helen Brown, Georgia School of Technology

Maslenikov, Mrs. Emily L., University of California, Berkeley

Mayhew, Isabel, University of Washington

Means, Frances Corry, University of Denver

Meckel, Clara Louise, Vanderbilt University

Meduri, Sarah Ernestine, White Plains, New York, Public Library

Meeks, James D., Yonkers Public Library

Meisel, Max, Hall-Mark Electrical Sales Co., Inc., Miami Beach, Florida

Mengel, Mildred M., Kutztown, Pennsylvania, State Teachers College

Ment, Bernadine, Detroit Public Library

Menzies, Elizabeth K., University of Washington

Merrill, IHallie Scott, Lees Junior College

Messler Mary J.. Trenton Public Library

Metcalf Milton C Brooklyn Public Library

Michael. Helen, College of St. Elizabeth

Miles, Gertrude E., Bucknell University

Miller, Gladys G., University of Minnesota

Miller, Mariana Kennedy, Columbia University

Miller, Mary Jo Evans. Austin College

Mitchem, Avis Dean, Fleming, Ohio

Modemann, Harriett Field, New London Junior College

Molenaer, Harriet, University of Texas
Montanari, Mrs. Phyllis Lee, c/o American Consulate,

Vitoria, E.S. Brazil

Moore, Betty Blaske, Catlin Memorial Library, Detroit

Moore, D. Eric, Lincoln University

Moore, Sister Esther Marie, Siena College

Moore Mildred Allen, Manhattan, Kansas, State College

Moran, Leila Padgett, Goucher College

Morgan, Whitney N.. B. T. Batsford, Ltd.

Morris, Sereta, S.C.A.P., C.I.\&E. Library, Kyoto, Honahu, Japan

Morris, True, University of Oregon

Morrow, Olive Irene, Fort Wayne Public Library

Moss, M. Eugenia, National College for Christian Workers

Mountain, Richard Allen, Caterpillar Tractor Co.

Muenscher, Elizabeth Anna, University of California, Berkeley

Mullenberg, Joan R., Ripon College

Mullman, Jeanne H., Oklahoma City University

Munro, Janet Elizabeth, Temple University

Murdock, Mrs. Mary, South Pasadena Public Library

Murphy, Mabel Ardis, Oklahoma College for Women

Murray, Thomas B., University of California, Berkeley

Murray, Virginia E., McGill University

Myers, Joseph H., Wilkes College

Myers, Lenore Miller, Bluffton College

Nemenzo, Mrs. Catalina A., Cebu College Library

Nemoy, Leon, Yale University

Nichols, Gladys, Urbana Junior College Institute of

Noone, Isabell Faith, Massachusetts

Technology

Nott, Mrs. Goldie Tilman, Ferris Institute

Odell, Gertrude Deirdre, San Bernardino Valley College

Oellrich, Gertrude L., Newark Public Library

Oldfather, Margaret, University of Illinois

Oliver, Rosa Virginia, Marshall College

Olney, Eleanor, Ohio State University

Olney, Eleanor, Ohio State University Norfolk

Orin, Leonard A.. Alabama Polytechnic Institute

Ostrander, Evah İ., Chicago Theological Seminary

Ostvold, Harald, Northwestern University

Owen, Grace Arlington, San Diego Public Library

Owen, Margaret, University of New Hampshire

Panghorn, Mark W., Jr., U. S. Geological Survey

Partridge, Frances Jane, Washington, D.C. Public Li brary

Paulin, Alice Davidson, American Steel \& Wire Research I-aboratory

Pearson. Barbara Elliott, Smith College

Pease, Margaret Gertrude, Whitworth College

Peerson, Ethel, Kingsport Public Library

Pehansek, Elizabeth V., University of Minnesota

Peirce, Billie Jo (Lochiridge), Texas A \& M College

Pendell, Iucille H., Gallaudet College

Penley, Mrs. Lavina Kirkman, California State Poly. technic College

Penrose, Charles, Bethany College

Peskind, Ira James, Wright Junior College

Peters, Frances Elizabeth, Free Library of Philadelphia

Peters, Wilk Smith, Tuskegee Institute

Petersem, Agnes J.; Feature Dept., Milwankee Journal, Milwaukee, Wisconsin

Peterson, Odrun E., Gustavus Adolphus College

Petronia, Sister Mary, Madonna College

Pezuela Ferrera, Manuel Gomez, Mexico City, Mexico

Phelps, Dorothy Jayne, University of Utah

Phipps, Barbara Helen, Emmanuel Missionary College

Pierce, Dorothy E., College of W \& M, V.P.T.. Norfolk

Plaut, Berta Gertrud, Congregation Emanuel, New York City

Plummer, Julia W., Lamar College

Plummer, Mary Ann, Bucknell University

Poland, Robert Ralph, University of Illinois

Poole, Mary Elizabeth, North Carolina State College, Raleigh

Pouncey, Lorene, Henry E. Huntington Library, San

Marino, Calif

Prince, Evelyn H., University of Chattanooga

Prince, Vivian C., University of Florida

Protzman, Ruth Marie, University of Oregon

Provendter, Kathryn, Pace Institute

Quinan, Jean Fuller, Stanford University 
Rackstraw, Elsie, Library of Congress

Randall, Paul L., Western Michigan College

Rasmussen, Sigurd Hartz, United Nations Library

Reed, Melia Randall, Chicago College of Osteopathy

Reed, Nanette, Michigan State College, East Lansing

Reiners, Thomas V., Manhattan College

Remley, Ralph Day, U. S. Civil Service Commission

Rendell, Mary Diana, University of British Columbia

Reparata, Sister Mary, Rosary College

Rettig, Mrs. Cleveland B. G., Florida Southern College

Reynolds, Helen Margaret, Úniversity of Illinois

Rice, Nannie H., Mississippi State College

Rich, Mrs. Annie B. F., Nathan and Henry B. Cleaves Law Library, Portland, Maine

Richard, Elizabeth, Kansas State College

Riddagh, Dorothy Jane, Albright College

Riddle, Margaret Selman, Ouachita College

Rieru, June, New Jersey Agricultural Experiment Station

Rise, Bernice M., University of Oregon

Roberts, Olive L., Northwestern State College, Natchitoches, La.

Robertson, Eleanor M., University of Illinois Robertson, Florence R., Danville (Virginia) Public
Library

Robertson, Ida Forbes, Iowa State College

Robinson, Dorothy Steckler, Southeastern College, Hanımond, La.

Rodabaugh, Mrs. Edna H., Purdue University

Rodell, Elizabeth G., Rice Institute

Rollins, Ruth Alice (Mrs. Dean B.), Institute of Paper Chemistry

Root, Elizabeth de W., Hartford Seminary Foundation

Rose, Mary Alice, Loyola College (Baltimore)

Ross, Marian, Louisiana State College

Rothstein, Samuel, University of British Columbia

Rowe, Helen Frances, University of Denver

Rowe, John R., Encyclopaedia Britannica

Rowell, Gordon A., Brooklyn College

Russell, Anna Loe, George Peabody College

Rystrom, Luella S., University of Michigan

Sachs, Judith Eve, Princeton Institute for Advanced Study

Saeger, John, Oberlin College

Sanders, Lvlah A., Rolvaag Memorial Library, Northfield, Minn.

Sanford, Dorothy Louise, Dillard University

Sanford, Frances E., Providence Public Library

Saupe, Frances Helen, Oklahoma A \& M College

Sayer, Edith Angie, Truett-McConnell Junior College

Schilpp, Emily C., Johns Hopkins University

Schimansky, Helene Elizabeth, University of California, Los Angeles

Schuler, Eric T., Library of Congress

Schlueter, Reinhold A., University of Wisconsin

Schlutie, Lorraine, Pratt Institute

Schuerman, Rev. C. J., Saint Joseph's College

Schweickart, Ruth L., Miami University

Schweikart, Helen L., Huntington, W.Va

Scott, Walter B., Alabama Polytechnic Institute

Scranton, Mrs. Faith Burnett, Stephens College

Scriven, Margaret, Chicago Historical Society

Secor, Jane Curtis, Denison University

Segan, Mildred Marie, University of Notre Dame

Sewell, Mary Robert, University of North Carolina

Shankroff, Dorothy, University of California, Berkeley

Shannon, Dwight W., Texas A \& M College

Sharp, Camilla, Henderson State Teachers College, Arkadelphia, Ark.

Sheffield, Pyrrha Buchner, Portland Cement Association Library

Sheppard. Edward Lee, University of Illinois

Shields, Ethel A., Eastman Kodak Business Library

Shine, Lou Sullivan, University of North Carolina

Shipley, Oliver M., U. S. Dept. of Agriculture

Shreve, Emily Gray, Dept. of Army, Office of Chief of Transportation

Sieving, Hilmar A., University of Chicago

Simmons, Mildred, Lexington, Kentucky

Simpson, Mrs. Beth A.. Medical Field Service School, Fort Sam Houston, Tex.

Sioussat, St. George L., Library of Congress

Sivertz, Chloe Thompson, University of Washington

Skinner, Mrs. Olive D., Madison, Wisconsin

Skofield. Mrs. Harriet Ćlark, University of Florida

Smith, Betty Clem, Hawaiian Economic Foundation

Smith, Diana C., Armed Forces Staff College, Norfolk
Smith, Ella Frances, University of Virginia

Smith, Martha Frances, Florida State University

Smith, Sidney Butler, University of Vermont

Smith, Stewart Peden, University of North Carolina

Smogard, Elaine Carol, Beloit College

Snavely, Virginia Graham, Ohio State University

Snead, Marie Elverna, Ambridge, Pennsylvania, Junior

High School

Snider, Winifred Helen, Lake Erie College

Snyder, Maye E., Pennsylvania State Library

Southworth, June C., Ohio University

Spartz, Arlyn Mary, Marquette University

Spence, Elizabeth Henke, Beloit Public Library

Spengler, Alice Louise, Colorado Woman's College

Spofford, Walter R. University Club of Chicago

Sprague, Morteza Drexel, Tuskegee Institute

Sprow, Allen Jenkins, Brown University

Sprug, Joseph William, Catholic University

Stahlecker, Luanna H., University of Iowa

Stalker, Frances, Indianapolis Public Library

Stanford, Spencer C., Brookhaven National Laboratory, Upton, New York

Stapleton, Margaret L., Tacoma Public Library

Steele, Marion D., Library of Congress

Steeves, H. Alan, Museum of American Numismatic Society

Stewart, Frederic W., Hunter College

Stewart, Harriett W., Army Information School, Carlisle, $\mathrm{Pa}$

Stickle, Nellie R., Elmhurst College

Stirling, Margaret, Army Medical Library, Washington, D.C.

Stockert, Helen, West Virginia Wesleyan College

Stokes, Katharine M., University of Illinois

Stone, Evelyn P., U.'S. Dept. of Agriculture

Stones, Mrs. Elleine H., Detroit Public Library

Stover, Webster, American Librarians' Agency

Stowe, Fern Elizabeth, New Paltz, New York, State Teachers College

Studer, Charlotte, Michael Reese Hospital, Chicago

Sturtevant, Anne F., University of Iowa

Suartz, Beatrice M.,' Wellesley College

Sullivan, Lola E., University of Missouri

Sullivan, Thomas Edward, Yale University

Sumner, Oween, Florida Southern College

Swain, Olive, California State Library

Swem, Earl Gregg, William and Mary College

Swift, Emma Belle, Rochester Public Library

Syler, Rosa Mae, Lamar College

Tangen, May, University of Iowa

Tapper, Ethel W., Aurora College

Taube, Mortimer, Library of Congress

Taylor, Lucille G., Washington University

Taylor, Mary D., Texas State College for Women

Thayer, Frances Theodora, University of California, Berkeley

Thayer, Mrs. Lewis A., Lewis and Clark Library, Portland, Oregon

Thickman, Mrs. Muriel H., College of the City of New York

Thomas, J. Luther, Florida Agric. and Mech. College

Thomas, Martha Reynolds, Columbia University

Thomen, Harold Ordell, Library of Congress

Thompson, John Richard, Indiana University

Thompson, Margaret W., U. S. Information Service

Thumm, Janice Levaine, Baidwin-Wallace College

Ticknor, William Edward, Enoch Pratt Free Library

Tierney, Jessie N., Chadbourne, Wallace, Parke \&

Whiteside, New Ÿork, N.Y.

Tiffany, Ellen M., Pennsylvania State College, Schuylkill Undergraduate Center

Tisdel, Kenneth S., University of Missouri

Tish, Lois, Cascade College

Tjaden, Priscilla Charlotte, University of Kansas

Todd-Davis, Elmyra R., Langston University

Tolman, Lorraine E., Worcester, Mass., State Teachers College

Toth, Mrs, Margaret K., Women's College Library, Rochester, New York

Trammell, Mary Pauline, University of Florida

Trautman, Ray L., New York, N.Y.

Trelease, Robert J., Remington Rand, Inc.

Trevillian, Mrs. Bernice S., Phoenix College

Tucker, Cynthia Furneaux, Amherst College

Tucker, Elinor Brown, University of Iowa

Tucker, Rosalie G., Vassar College

Tuffley, Anna Frances, Northern Michigan College of 
Education

Tullis, Isabel B., Central Washington College of Education

Tunnell, Mrs. Enrica, Columbia Unjversity

Turner, Justin G., El Royale Library, Los Angeles

Uhr, Frances, U. S. Immigration and Naturalization Service, Philadelphia

Valldejuli, Maria A., University of Puerto Rico

Vander Ploeg, Jeannette, San Jose, California, State College

Van Horne, Bernard, John Crerar Library, Chicago Van Norman, C. Elta, Geneseo, New York, State Teachers College

Van Zee, Gertrude M., Kalamazoo College

Verghese, P. Joseph, Dept. of Agriculture, Malayan Union

Vernon, Daisy May, Wichita University

Vognild, Evelyn S., University of Denver

Walker, E. Virginia, Swarthmore College

Walker, Lois Janet, Pacific Union College

Walkinshaw, Mildred Frances, Sidney Blumenthal \& Co., Inc., Shelton, Conn.

Wallace, Mrs, M Isabella B. Whiting, Indiana

Walsh, Bertrand McK., New York Public Library

Ward, Elizabeth Willingham, Lakewood Public Library

Warner, Sam B., Library of Congress

Webb, David A., Northwestern University

Weedon, Pauline 1)., Tampa Public Library

Wefel, Emelia Elizabeth, Cleveland Public Library

Wegner, Mary Jean, North Central College, Naper ville, Illinois

Welbes, Rev. Crispin, Quincy College

Weld, Eleanor Virginia, Princeton University

Welle, Edith Louise, University of Denver

Wellman, Murrell C., American Dental Association

Welsh, Doris V., Newberry Library, Chicago

Welsh, Dorothea, Winfield, Kansas, Southwestern College

Wember, Bertha C., Brooklyn College

Wenzel, Caroline, California State Library

West, Mary Ellen, Schenectady Public Library

West, Norbert Dick, Wayne University

Western, Mrs. Ida B., Simmons College

Wheeless, Estelle, Du Pont Technical Library, Wil mington

Whetstone, Vane Rea, Carnegie Library, Knoxville

White, Gretchen, College of Wooster
White, Viola Chittenden, Middlebury College

Whitehead, Olive F., American Sugar Refining Co., Philadelphia

Whitenack, Arthur Elmer, Mary Washington College

Whitman, Davis, University of Mississippi

Whitman, Ruth, North Dakota Agricultural College

Wilder, Annette B., M.S.C. Demonstration School, Hattiesburg, Miss.

Wilkins, Frances Vroman, Keuka College

Williams, Marie B., Chicago Public Library

Williams, Virginia, University of New Mexico

Williamson, Mrs. Jeanette G., Sampson College

Williamson, William Landram, Baylor University

Willingham, John R., East Texas State Teachers College

Willoughby, Mabel E., Howard College

Wilson, Mabel Zoe, Western Washington College of Education

Wilson, Rebecca, University of Michigan

Winchester, Genevieve E., University of Wisconsin

Winer, Mrs. Monalee Ellis, Dept. of State, Washington, ID.C.

Wissler, Mary V., American Museum of Natural History

Wistrup, Ann Marie, Rensselaer Polytechnic Institute

Withenow, Lois Esther, Stockton Public Library

Wolfard, Nancy S., Queens College

Wood, Mrs. Chloe M., University of Southern California

Wood, Lulu L., Ashland College

Wood, Mrs. Rachel F., Mt. Vernon Seminary

Woodward, Anne M., Mary Baldwin College

Wright, Emma G., University of Oregon

Wuertz, Mrs. Clara Lorelle, University of Wichita

Wynne, Marjorie Gray, Yale University

Yager, Athol, University of Texas

Yelland, Philippa I., Michigan State College, East I ansing

Yen, Wen-Yu, National Roosevelt Library, Chungking

Yerkes, Helen Hay, Columbia University

Young, Dorothy B., Texas Technological College

Young, Elma I. Clark, Placer College

Young, Mrs. Gladys (Driver), Bendix Products Division, South Bend, Indiana

Zabriskie, Mary E., Radio Corporation of America, Lancaster, $\mathrm{Pa}$.

Zeides, Solomon, Yeshiva College

Zimmerman, Lee Franklin, University of Idaho

Zumstein, Marjorie Anne, University of Chicago

\section{Committee on Implementation of the National Plans}

The Committee on Implementation of the National Plans, recently authorized by the A.L.A. Executive Board and organized during the midwinter meeting, is now at work. Under the chairmanship of Edward A. Chapman, Rensselaer Polytechnic Institute Library, the C.I.N.P. is charged with seeing that something is done about the national plans which have been completed for every type of library service in the country. The mimeographed list of national plans, "Library Planning and Standards" (revised Jan. 1948) is available upon request from A.L.A. Headquarters.

The committee's over-all objective is to assist in the development and execution of plans for all types of library service in every state as a unified national effort, by all librarians working together, instead of along separate lines of special interest. As the first order of business, the committee is proposing a 1948 model state planning committee for libraries of the character indicated in the following statement of the committee's basic function:

To cause and assist the organization, in every state, of state planning committees for libraries so that they uniformly represent the interests of all types of libraries and library services at the same time, and include all outside representation that can conceivably contribute to or affect library planning and plan execution.

The work of C.I.N.P. is directed toward the achievement of one of the important elements of the A.L.A.'s Four Year Goals"... the further development of state plans within the framework of the national plans for all types of libraries." 


\section{Publications Committee to Assemble Significant Documents on Library Administration}

The following letter and checklist recently sent to librarians of college, university, and research libraries by the Publications Committee of A.C.R.L. inaugurates a project to assemble at A.L.A. Headquarters a comprehensive collection of source materials for the study of libraries in the sphere of interest represented by A.C.R.L.

The letter is self-explanatory. If your library did not receive a copy of this letter it is hoped that you will consider this as a request for the material described.

The Publications Committee of the Association of College and Reference Libraries needs your help in a plan to assemble in the office of the Executive Secretary of A.C.R.L. a collection of significant documents refiecting current practices in the administration of college, university, and research libraries.

The decision to undertake this project was dictated by the desire to make generally available to all interested libraries and library schools the wealth of information and experience recorded in documents now available only through individual search and contact with individual institutions more or less on a trial and error basis. It is the further hope of the committee that the existence in one place of a comprehensive collection of such materials will stimulate the production of useful articles and publications in this field. Specifically, the materials assembled from this request will be analyzed to determine the practicability of compiling a sourcebook for college and university library administration.

The plan, as now envisaged, provides for consultation of these materials at A.L.A. Headquarters, the loan of the materials to libraries, and possible reproduction for use of libraries and library training agencies.

For your convenience, a checklist of the types of material wanted is presented below. In general, the aim of the project is to bring together in one place not only the published materials such as library handbooks, personnel codes, statutes relating to the library, rules, regulations, and loan privileges, but also manuscript materials such as budgets and budget justifications, letters and memoranda prepared for policy conferences with library staff or institutional officers and faculty, and memoranda or orders issued to implement policy decisions. Confidential or semiconfidential material could be labeled so that it would not be released except by permission of the originating institution.

To assure the continued success of this project, it is requested that you not only supply materials currently available but that you continue to send without further solicitation similar materials as they become available.

Any questions regarding interpretation of this request or regarding use of the material should be sent to N. Orwin Rush, Executive Secretary, A.C.R.L., American Library Association, 50 E. Huron St., Chicago II, who will either supply the information desired or forward correspondence to the Publications Committee for reply.

Your cooperation in this project is earnestly solicited.

Richard H. Logsdon, Chairman

A.C.R.L. Publications Committee Columbia University Library

\section{Checklist of Materials Desired}

Annual reports

Staff manuals

Student handbooks containing library data

Faculty handbooks

Library news letters and book lists containing news items

Statement of rules, regulations, loan privileges

Budget and budget justifications

Personnel codes

Classification and pay plans

Statutes related to the library

Interpretations of statutes

Organization charts

Statements describing book selection policies

Departmental manuals such as acquisitions, cataloging, binding and interlibrary loan

Building plans

General orders

Statements covering organization, functions 
or responsibilities of the library or any of its departments

Letters, memoranda, or other documents prepared for policy meetings with library staff, institutional officials, library committees, etc.

Letters, memoranda, or other documents issued to implement policy decisions

Statements of responsibility or functions of library committee or other advisory groups

Reports of surveys including self-surveys

Documents describing organization and activi- ties of staff associations, etc.

Statements describing local cooperative agreements regarding book acquisition, service, etc.

Documents describing local agreements for exchange of catalog cards and establishment of union catalogs (particularly those not generally known to the profession)

Documents describing "Friends of the Library" and similar activities

Documents describing extent of staff participations in management of the library.

\section{New Program at Columbia}

W ITH the winter session of $1948-49$, the School of Library Service, Columbia University, inaugurates two completely new programs of study leading to the graduate degrees of master of science and doctor of philosophy. This is the first basic revision of the program and degree structure since the school was reorganized in 1926.

The content of the curriculum for the master's degree may be described under the four headings of foundations, resources, readers, and methods, and consists of ten threepoint required courses, and an additional six points taken from the elective offerings of the school or other departments of the university.

The doctoral program of instruction for 1948-49 will offer a series of seminars in foundations of librarianship, bibliographical re- search, library resources, readers and reading, organization of materials, library administration, and education for librarianship.

The minimum period of residence for the master's program is a winter, spring, and summer session. For those students who cannot carry the expense of a full-time study program, the work-study program offers a half-time position in a college, research, or public library to be combined with a semester study program of nine hours taken for four consecutive winter and spring sessions. Income during the winter months ranges from $\$ 85$ to $\$ 100$ with a double salary during the summer months.

Application for admission to all courses must be made on forms obtainable from the Office of University Admissions or the School of Library Service, Columbia University. 